\title{
New results about set colorings of graphs
}

\author{
J.P. Boutin \\ Département Informatique, I.U.T. Lyon 1, France \\ jean-pierre.boutin@univ-lyon1.fr \\ E. Duchêne, B. Effantin, H. Kheddouci, H. Seba \\ Laboratoire GAMA, \\ Université Claude Bernard Lyon 1, Université de Lyon, F-69622 France \\ eric.duchene@univ-lyon1.fr, brice.effantin-dit-toussaint@univ-lyon1.fr \\ hamamache.kheddouci@univ-lyon1.fr, hamida.seba@univ-lyon1.fr
}

Submitted: Oct 7, 2009; Accepted: Nov 26, 2010; Published: Dec 10, 2010

Mathematics Subject Classification: 05C15

\begin{abstract}
The set coloring problem is a new kind of both vertex and edge coloring of a graph introduced by Suresh Hegde in 2009. Only large bounds have been given on the chromatic number for general graphs. In this paper, we consider the problem on paths and complete binary trees, and show that it can be reduced to the computation of a transversal in a special Latin square, i.e., the XOR table. We then investigate a variation of the problem called strong set coloring, and we provide an exhaustive list of all graphs being strongly set colorable with at most 4 colors.
\end{abstract}

\section{Introduction}

We choose standard notations and definitions in graph theory. If $G=(V, E)$ is a given graph, the order of $G$ is the number of vertices $|V|$, and the size of $G$ is the number of edges $|E|$. If $u, v$ are two vertices of $V$, we denote by $u \sim v$ the fact that $u$ and $v$ are adjacent in $G$. We will consider simple connected graphs in the whole paper. Moreover, the cardinality of a set $S$ will be denoted by $\# S$.

The notion of set coloring of a graph was introduced in 2009 by Hegde [4]. In its original version, both vertices and edges of an undirected graph are colored with finite sets of positive integers. The color of an edge $(u, v)$ is given by the symmetric difference of the colors of $u$ and $v$. A graph is said to be set colorable if there exists an assignment of colors on the vertices such that both conditions are fulfilled:

(i) all the colors on the vertices are distinct,

(ii) all the colors on the edges are distinct. 


\begin{tabular}{c|cccccccccccccccc}
$\oplus$ & 0 & 1 & 2 & 3 & 4 & 5 & 6 & 7 & 8 & 9 & 10 & 11 & 12 & 13 & 14 & 15 \\
\hline 0 & 0 & 1 & 2 & 3 & 4 & 5 & 6 & 7 & 8 & 9 & 10 & 11 & 12 & 13 & 14 & 15 \\
1 & 1 & 0 & 3 & 2 & 5 & 4 & 7 & 6 & 9 & 8 & 11 & 10 & 13 & 12 & 15 & 14 \\
2 & 2 & 3 & 0 & 1 & 6 & 7 & 4 & 5 & 10 & 11 & 8 & 9 & 14 & 15 & 12 & 13 \\
3 & 3 & 2 & 1 & 0 & 7 & 6 & 5 & 4 & 11 & 10 & 9 & 8 & 15 & 14 & 13 & 12 \\
4 & 4 & 5 & 6 & 7 & 0 & 1 & 2 & 3 & 12 & 13 & 14 & 15 & 8 & 9 & 10 & 11 \\
5 & 5 & 4 & 7 & 6 & 1 & 0 & 3 & 2 & 13 & 12 & 15 & 14 & 9 & 8 & 11 & 10 \\
6 & 6 & 7 & 4 & 5 & 2 & 3 & 0 & 1 & 14 & 15 & 12 & 13 & 10 & 11 & 8 & 9 \\
7 & 7 & 6 & 5 & 4 & 3 & 2 & 1 & 0 & 15 & 14 & 13 & 12 & 11 & 10 & 9 & 8 \\
8 & 8 & 9 & 10 & 11 & 12 & 13 & 14 & 15 & 0 & 1 & 2 & 3 & 4 & 5 & 6 & 7 \\
9 & 9 & 8 & 11 & 10 & 13 & 12 & 15 & 14 & 1 & 0 & 3 & 2 & 5 & 4 & 7 & 6 \\
10 & 10 & 11 & 8 & 9 & 14 & 15 & 12 & 13 & 2 & 3 & 0 & 1 & 6 & 7 & 4 & 5 \\
11 & 11 & 10 & 9 & 8 & 15 & 14 & 13 & 12 & 3 & 2 & 1 & 0 & 7 & 6 & 5 & 4 \\
12 & 12 & 13 & 14 & 15 & 8 & 9 & 10 & 11 & 4 & 5 & 6 & 7 & 0 & 1 & 2 & 3 \\
13 & 13 & 12 & 15 & 14 & 9 & 8 & 11 & 10 & 5 & 4 & 7 & 6 & 1 & 0 & 3 & 2 \\
14 & 14 & 15 & 12 & 13 & 10 & 11 & 8 & 9 & 6 & 7 & 4 & 5 & 2 & 3 & 0 & 1 \\
15 & 15 & 14 & 13 & 12 & 11 & 10 & 9 & 8 & 7 & 6 & 5 & 4 & 3 & 2 & 1 & 0
\end{tabular}

Figure 1: The XOR table $A_{4}$.

In this paper, we choose to consider the problem in another but equivalent way, which appears to us more natural. Stronger reasons of this choice will be proposed in section 2 . If $n$ is a positive integer, denote by $Z_{n}$ the set $\left\{0,1, \ldots, 2^{n}-1\right\}$ of the $2^{n}$ first nonnegative integers. For any two values $i, j \in Z_{n}$, we denote by $i \oplus j$ the $X O R$ value (also known as Nim-sum or addition in binary without carries.) It is well known that $\left(Z_{n}, \oplus\right)$ is a finite group. We denote by $A$ the semi-infinite matrix defined by $A(i, j)=i \oplus j$ for $i, j \in \mathbb{Z}_{\geq 0}$, and $A_{n}$ the finite matrix consisting of the first $2^{n}$ rows and first $2^{n}$ columns of $A$. Figure 1 gives the computation of $i \oplus j$ with $i, j \in Z^{4}$, i.e., the matrix $A_{4}$.

Definition 1. A Latin square over an alphabet $\alpha_{p}$ of size $p>0$ is a $p \times p$ table filled with the $p$ different symbols of $\alpha_{p}$ in such a way that each symbol occurs exactly once in each row and exactly once in each column.

As defined in [3], for every positive integer $n$, the matrix $A_{n}$ is closed n-nim-regular, implying that it is a Latin square over $Z_{n}$. Sudoku grids are other examples of Latin squares.

Given a graph $G=(V, E)$ and a positive integer $n$, we define a function $f_{n}: V \rightarrow Z_{n}$ which assigns to each vertex of $v \in V$ a color $f(v)$. We also define a function $f_{n}^{\oplus}: E \rightarrow Z_{n}$, which assigns colors to the edges, and defined as $f_{n}^{\oplus}(u, v)=f_{n}(u) \oplus f_{n}(v)$ for all $(u, v) \in E$.

A graph is said to be $n$ set colorable if there exist two functions $f_{n}$ and $f_{n}^{\oplus}$ that are injective. Figure 2 shows a 4 set colorable graph. In view of its size $\left(|E|=10>2^{3}\right)$, we can also assert that the set coloring number of this graph is equal to 4 .

In his paper [4], Hegde introduces three problems related to the set coloring:

- The determination of the set coloring number $\sigma(G)$ of a graph $G$, which is the 
smallest $n$ such that $G$ is $n$ set colorable.

- The strong set coloring problem. A graph $G=(V, E)$ is said to be strongly $n$-set colorable if there exists a $n$ set coloring such that $f_{n}(V) \cup f_{n}^{\oplus}(E)$ make a partition of $Z_{n} \backslash\{0\}$. Note that if such a coloring exists, then we necessarily have $|V|+|E|=$ $2^{n}-1$. Figure 2 gives an example of a strongly 4 -set colorable graph.

- The proper set coloring problem. A graph $G=(V, E)$ is said to be proper set colorable if it is set colorable with $f_{n}^{\oplus}(E)=Z_{n} \backslash\{0\}$. The existence of a proper coloring implies the equality $|E|=2^{n}-1$.
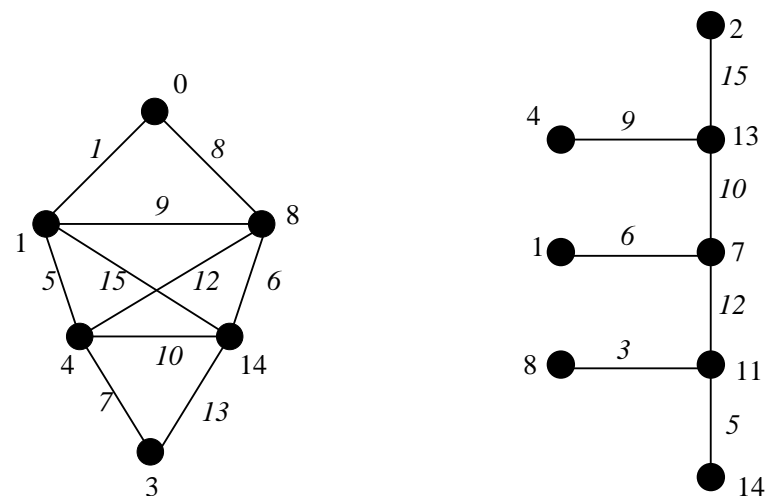

Figure 2: Examples of a 4 set colorable graph (on the left) and a strongly 4-set colorable graph (on the right)

This paper is organized as follows: in section 2, we consider the basic set coloring problem and the computation of $\sigma(G)$ on special families of graphs such as paths and complete binary trees. For both cases, we show that an optimal set coloring can be obtained by computing a certain type of transversal in the XOR table (the definition of a transversal in a Latin square will be given in section 2). In section 3, we give new necessary conditions for a graph to be strongly set colorable. As an application of these conditions, we provide an exhaustive list of the graphs that are strongly 3- and 4-set colorable.

\section{Set coloring number}

In his paper [4], Hegde especially focused on the strong and the proper variants of the set coloring problem. Investigations on the set coloring number were not considered, except lower and upper bounds in the general case. Indeed, it was straightforwardly said that for any graph $G=(V, E)$, we have

$$
\left\lceil\log _{2}(|E|+1)\right\rceil \leq \sigma(G) \leq|V|-1
$$

and the bounds are the best possible. In the current paper, we consider two families of graphs for which the lower bound may be tight: paths and complete binary trees. 
Before proceeding to the resolution, we investigate a connected problem derived from combinatorial number theory.

\subsection{A connected problem: finding a transversal in a Latin square over $Z_{n}$}

We here recall the definition of a transversal in a Latin square, which is due to Euler in 1779 .

Definition 2. A transversal $T$ in a $p \times p$ Latin square $L$ over $\{0, \ldots, p-1\}$ is a set of $p$ cells $\left\{L\left(i_{1}, j_{1}\right), \ldots, L\left(i_{p}, j_{p}\right)\right\}$ with $i_{k}, j_{k} \in\{0, \ldots, p-1\}$ for $k=1 \ldots p$, such that $\left\{i_{1}, \ldots, i_{p}\right\}=\left\{j_{1}, \ldots, j_{p}\right\}=\left\{L\left(i_{1}, j_{1}\right), \ldots, L\left(i_{p}, j_{p}\right)\right\}=\{0, \ldots, p-1\}$. In other words, $T$ has exactly one cell in each row, one in each column, and the cells contain all the symbols of $\{0, \ldots, p-1\}$.

For convenience for the reader, a transversal will be denoted as a set of triplets $\left\{\left(i_{1}, j_{1}, L\left(i_{1}, j_{1}\right), \ldots,\left(i_{p}, j_{p}, L\left(i_{p}, j_{p}\right)\right\}\right.\right.$.

There are few results guaranteeing the existence of a transversal in Latin squares. One can mention two important conjectures that are still open. In $[1,5]$, Ryser conjectured that any Latin square of odd size admits a transversal. Later, Brualdi [2] made the conjecture that any Latin square of size $n$ has a partial transversal of size at least $n-1$, where a partial transversal is a subset of a transversal.

In the context of set coloring, we are interested in the Latin square $A_{n}$ of the XOR operator. We will prove that we can guarantee the existence of a transversal for each value of $n \geq 2$. Moreover, we provide a recursive algorithm that builds such a transversal.

Proposition 1. For every positive integer $n \geq 2$, the Latin square $A_{n}$ admits a transversal.

Proof. We consider the following recursion hypothesis:

$\left(H_{n}\right)$ : The table $A_{n}$ admits a transversal $T_{n}$ such that for all $0 \leq j<2^{n}$, the cells $(i, j, i \oplus j)$ and $\left(i^{\prime}, j \oplus 1, i^{\prime} \oplus j \oplus 1\right)$ satisfy $i \oplus i^{\prime} \geq 2^{n-1}$. In other words, if we consider the cells of the $j^{\text {th }}$ and the $(j \oplus 1)^{\text {th }}$ columns, one of both has a value less than $2^{n-1}$, while the other has a value greater or equal to $2^{n-1}$.

One can check that $A_{2}$ with the following transversal satisfies $\left(H_{2}\right)$ :

$$
(0,0,0),(2,3,1),(3,1,2),(1,2,3)
$$

Now assume that $\left(H_{n-1}\right)$ is true for some transversal $T_{n-1}$ with $n>2$, and consider the table $A_{n}$. The set $T_{n}$ is built as follows, divided into 4 types of cells:

1. The cells $(i, j, i \oplus j)$ of $T_{n-1}$ satisfying $0 \leq i \oplus j<2^{n-2}$ also belong to $T_{n}$.

2. For each cell $(i, j, i \oplus j)$ of $T_{n-1}$ satisfying $2^{n-2} \leq i \oplus j<2^{n-1}$, add the cell $(i+$ $\left.2^{n-1}, j+2^{n-1},\left(i+2^{n-1}\right) \oplus\left(j+2^{n-1}\right)\right)$ to $T_{n}$. Since $0 \leq i, j<2^{n-1}$, those cells can also be written as $\left(i+2^{n-1}, j+2^{n-1}, i \oplus j\right)$. 
3. For each cell $(i, j, i \oplus j)$ of $T_{n-1}$ satisfying $0 \leq i \oplus j<2^{n-2}$, add the cell $\left(i+2^{n-1}, j+\right.$ $\left.1,\left(i+2^{n-1}\right) \oplus(j+1)\right)$ to $T_{n}$ if $j \equiv 0(2)$, and the cell $\left(i+2^{n-1}, j-1,\left(i+2^{n-1}\right) \oplus(j-1)\right)$ otherwise. Since $0 \leq i, j<2^{n-1}, j+1=j \oplus 1$ if $j \equiv 0(2)$ and $j-1=j \oplus 1$ if $j \equiv 1(2)$, then those cells can be written as $\left(i+2^{n-1}, j \oplus 1,\left(i \oplus j \oplus 1+2^{n-1}\right)\right)$.

4. For each cell $(i, j, i \oplus j)$ of $T_{n}$ of type 2 (i.e., satisfying $2^{n-1} \leq i, j<2^{n}$ ), add the cell $\left(i-2^{n-1}, j+1,\left(i-2^{n-1}\right) \oplus(j+1)\right)$ to $T_{n}$ if $j \equiv 0(2)$, and the cell $\left(i-2^{n-1}, j-\right.$ $\left.1,\left(i+2^{n-1}\right) \oplus(j-1)\right)$ otherwise. Those cells can be written as $\left(i-2^{n-1}, j \oplus 1,(i \oplus\right.$ $\left.\left.j \oplus 1+2^{n-1}\right)\right)$.

Figure 3 shows the localisation of the cells of $T_{n}$ according to their type, with the range of values they contain.

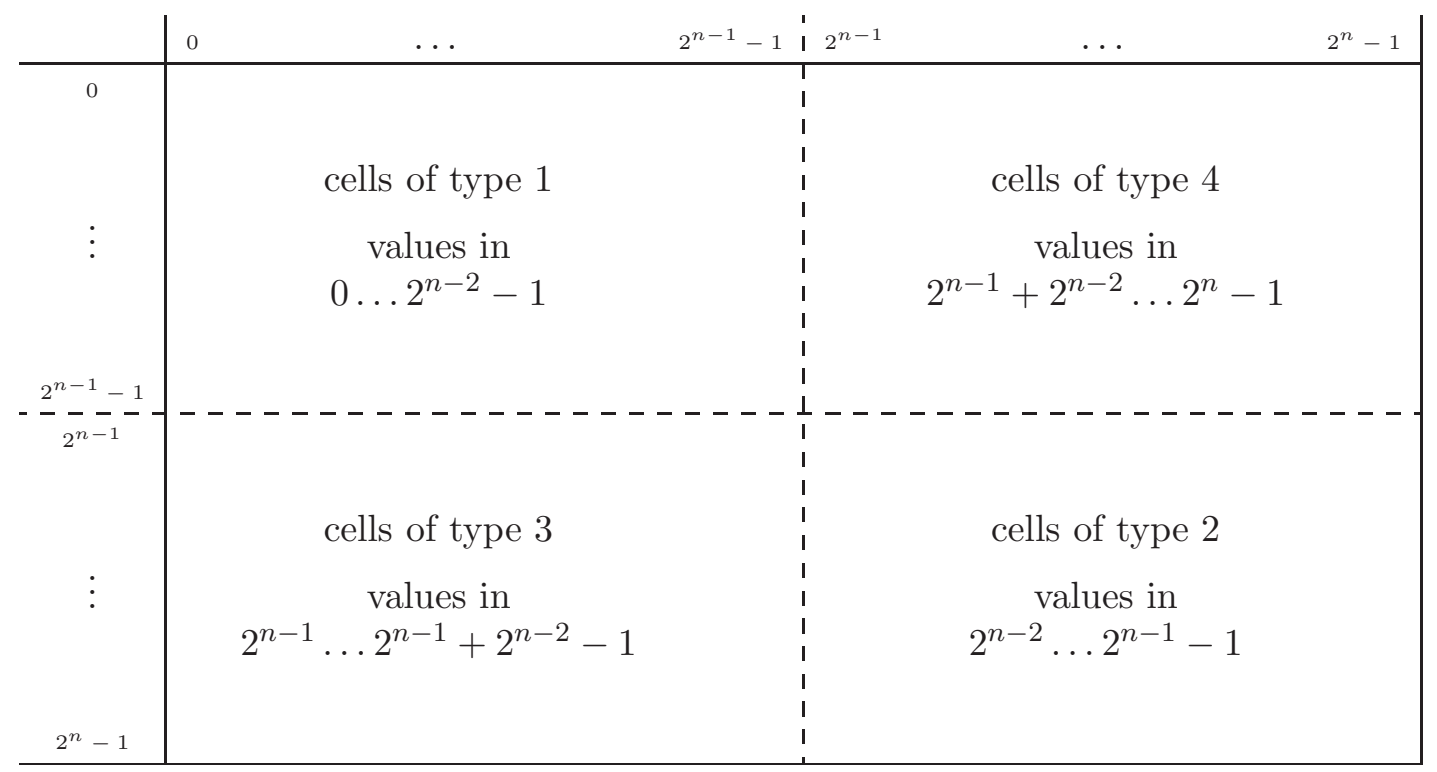

Figure 3: Types and values of the cells when building $T_{n}$ in the table $A_{n}$.

We now show that $T_{n}$ is a transversal of $A_{n}$. Since $T_{n-1}$ is also a transversal, the cells of type 1 and 2 contain all the values between 0 and $2^{n-1}-1$. For the same reason, for $0 \leq i \oplus j<2^{n-2}$, the values of the cells of type 3 take all the values $i \oplus j \oplus 1+2^{n-1}$, i.e., the set $\left\{2^{n-1}, \ldots, 2^{n-1}+2^{n-2}-1\right\}$. The cells of type 4 take all the values $i \oplus j \oplus 1+2^{n-1}$ for $2^{n-2} \leq i \oplus j<2^{n-1}$, i.e., they take all the values $2^{n-1}+2^{n-2}, \ldots, 2^{n}-1$. Collecting the values of the four types of cells, we obtain that $T_{n}$ contains all the values between 0 and $2^{n}-1$.

In order to show that there is no repetition of cell in a same column, first note that there cannot be two cells of the same type in the same column. Otherwise this would mean that it is also the case in $T_{n-1}$. There are only two possibilities for having two cells in the same column:

- A cell of type 1 is in the same column as a cell of type 3. According to the definition of $T_{n}$, there is a cell of type 1 in column $j$ iff there is a cell of type 3 in column $j \oplus 1$. 


\begin{tabular}{c|cc:cc}
$\oplus$ & 0 & 1 & 2 & 3 \\
\hline 0 & 0 & 1 & 2 & 3 \\
1 & 1 & 0 & 3 & 2 \\
\hdashline 2 & 2 & 3 & 0 & 1 \\
3 & 3 & 2 & 1 & 0
\end{tabular}

\begin{tabular}{c|cccc:cccc}
$\oplus$ & 0 & 1 & 2 & 3 & 4 & 5 & 6 & 7 \\
\hline 0 & 0 & 1 & 2 & 3 & 4 & 5 & 6 & 7 \\
1 & 1 & 0 & 3 & 2 & 5 & 4 & 7 & 6 \\
2 & 2 & 3 & 0 & 1 & 6 & 7 & 4 & 5 \\
3 & 3 & 2 & 1 & 0 & 7 & 6 & 5 & 4 \\
\hdashline 4 & 4 & 5 & 6 & 7 & 0 & 1 & 2 & 3 \\
5 & 5 & 4 & 7 & 6 & 1 & 0 & 3 & 2 \\
6 & 6 & 7 & 4 & 5 & 2 & 3 & 0 & 1 \\
7 & 7 & 6 & 5 & 4 & 3 & 2 & 1 & 0
\end{tabular}

Figure 4: The transversals $T_{2}$ (left) and $T_{3}$ (right).

Moreover, since the cells of type 1 satisfy $\left(H_{n-1}\right)$, there is no pair of cells of type 1 in two consecutive columns $(j, j \oplus 1)$.

- A cell of type 2 is in the same column as a cell of type 4. The argument is the same, since there is no pair of cells of type 2 in two consecutive columns $(j, j \oplus 1)$.

Note that the above argument also shows that given two consective columns $(j, j \oplus 1)$ of $A_{n}$ (for $0 \leq j<2^{n}$ ), each of them contains exactly either one cell of type 1 and one of type 3 , or one of type 2 and one of type 4 , which fulfills the condition of $\left(H_{n}\right)$.

We now prove that there is no repetition of cells in a same row. As for the columns, if it is the case, the cells must have different types:

- A cell of type 2 is in the same row as a cell of type 3 . Denote by $i$ the row index of these two cells of type 2 and 3 . Then $i-2^{n-1}$ is the index of a unique cell whose value $v$ both satisfies $0 \leq v<2^{n-2}$ (definition of type 3 ), and $2^{n-2} \leq v<2^{n-1}$ (definition of type 2). We get a contradiction.

- A cell of type 1 is in the same row as a cell of type 4. Denote by $i$ the row index of these two cells of type 1 and 4 . Then $i+2^{n-1}$ is the index of a cell of type 2 , whose value $v$ satisfies $2^{n-2} \leq v<2^{n-1}$ (definition of type 4 ). Hence $i+2^{n-1}-2^{n-1}=i$ is also the index of a cell with the value $v$, which is greater than a cell value of type 1. This concludes the proof.

Hence $T_{n}$ build in this way satisifies $\left(H_{n}\right)$.

Figures 4 and 5 illustrate the construction of the transversal $T_{n}$ satisfying $\left(H_{n}\right)$ for $n=$ $2,3,4$. The initialization is done with the transversal $T_{2}$ given in the proof of Proposition 1.

In addition to the basic definition of a transversal, our investigations of the set coloring problem on paths made us define the following constraint variant: 


\begin{tabular}{c|cccccccc:cccccccc}
$\oplus$ & 0 & 1 & 2 & 3 & 4 & 5 & 6 & 7 & 8 & 9 & 10 & 11 & 12 & 13 & 14 & 15 \\
\hline 0 & 0 & 1 & 2 & 3 & 4 & 5 & 6 & 7 & 8 & 9 & 10 & 11 & 12 & 13 & 14 & 15 \\
1 & 1 & 0 & 3 & 2 & 5 & 4 & 7 & 6 & 9 & 8 & 11 & 10 & 13 & 12 & 15 & 14 \\
2 & 2 & 3 & 0 & 1 & 6 & 7 & 4 & 5 & 10 & 11 & 8 & 9 & 14 & 15 & 12 & 13 \\
3 & 3 & 2 & 1 & 0 & 7 & 6 & 5 & 4 & 11 & 10 & 9 & 8 & 15 & 14 & 13 & 12 \\
4 & 4 & 5 & 6 & 7 & 0 & 1 & 2 & 3 & 12 & 13 & 14 & 15 & 8 & 9 & 10 & 11 \\
5 & 5 & 4 & 7 & 6 & 1 & 0 & 3 & 2 & 13 & 12 & 15 & 14 & 9 & 8 & 11 & 10 \\
6 & 6 & 7 & 4 & 5 & 2 & 3 & 0 & 1 & 14 & 15 & 12 & 13 & 10 & 11 & 8 & 9 \\
7 & 7 & 6 & 5 & 4 & 3 & 2 & 1 & 0 & 15 & 14 & 13 & 12 & 11 & 10 & 9 & 8 \\
\hdashline 8 & 8 & 9 & 10 & 11 & 12 & 13 & 14 & 15 & 0 & 1 & 2 & 3 & 4 & 5 & 6 & 7 \\
9 & 9 & 8 & 11 & 10 & 13 & 12 & 15 & 14 & 1 & 0 & 3 & 2 & 5 & 4 & 7 & 6 \\
10 & 10 & 11 & 8 & 9 & 14 & 15 & 12 & 13 & 2 & 3 & 0 & 1 & 6 & 7 & 4 & 5 \\
11 & 11 & 10 & 9 & 8 & 15 & 14 & 13 & 12 & 3 & 2 & 1 & 0 & 7 & 6 & 5 & 4 \\
12 & 12 & 13 & 14 & 15 & 8 & 9 & 10 & 11 & 4 & 5 & 6 & 7 & 0 & 1 & 2 & 3 \\
13 & 13 & 12 & 15 & 14 & 9 & 8 & 11 & 10 & 5 & 4 & 7 & 6 & 1 & 0 & 3 & 2 \\
14 & 14 & 15 & 12 & 13 & 10 & 11 & 8 & 9 & 6 & 7 & 4 & 5 & 2 & 3 & 0 & 1 \\
15 & 15 & 14 & 13 & 12 & 11 & 10 & 9 & 8 & 7 & 6 & 5 & 4 & 3 & 2 & 1 & 0
\end{tabular}

Figure 5: The transversal $T_{4}$.

Definition 3. Let $T$ be a transversal on a Latin square $L$ according to Definition 2. Let $\Gamma:\{0, \ldots, p-1\} \rightarrow\{0, \ldots, p-1\}$ be the function that associates to each row index $i$ the column index $\Gamma(i)$ such that $L(i, \Gamma(i))$ is a cell of $T$. The transversal $T$ is said to be a CP-transversal if $\Gamma$ is a circular permutation of size $p$ or $p-1$.

Conjecture 1. For every positive integer $n \geq 2$, the Latin square $A_{n}$ admits a CPtransversal.

We have checked the validity of Conjecture 1 until $n=5$. Unfortunately, the algorithm described in the proof of Proposition 1 does not always provide a CP-transversal. The transversal $T_{2}, T_{3}$ and $T_{4}$ of Figures 4 and 5 are CP-transversals. Some local "cells exchanges" are required to convert $T_{5}$ into a CP-transversal. However, we did not find deterministic rules to describe such transformations in the general case.

\subsection{Set coloring number of paths}

We first start by showing that in the case of a path of order $2^{n}$, the lower bound $\left\lceil\log _{2}(|E|+\right.$ $1)\rceil=n$ cannot be reached for $\sigma(G)$. However, we then prove that $\sigma(G)=n+1$.

Lemma 1. Given any positive integer $n \geq 2$, a path $P$ of order $2^{n}$ satisfies $\sigma(P)>n$.

Proof. Denote by $P_{1}, \ldots, P_{2^{n}}$ the vertices of $P$ such that $\left(P_{i}, P_{i+1}\right) \in E$ for all $1 \leq i \leq$ $2^{n}-1$. We clearly have that $\sigma(P) \geq n$. Now assume that there exists a set coloring $\left(f_{n}, f_{n}^{\oplus}\right)$ of $P$ with $n$ colors. Hence we have $\left\{f_{n}^{\oplus}\left(P_{i}, P_{i+1}\right): 1 \leq i \leq 2^{n}-1\right\}=Z_{n} \backslash\{0\}$ since the colors of the edges are all distinct. Therefore we get the following equality:

$$
f_{n}^{\oplus}\left(P_{1}, P_{2}\right) \oplus \ldots \oplus f_{n}^{\oplus}\left(P_{2^{n-1}}, P_{2^{n}}\right)=1 \oplus \ldots \oplus 2^{n}=0
$$


By definition of $f_{n}^{\oplus}$, we get

$$
f_{n}\left(P_{1}\right) \oplus f_{n}\left(P_{2^{n}}\right)=0
$$

This contradicts the injectivity of $f_{n}$.

Proposition 2. Given any positive integer $n \geq 2$, a path $P$ of order $2^{n}$ satisfies $\sigma(P)=$ $n+1$.

Proof. From Lemma 1, it now suffices to show that there exists a set coloring of $P$ with $n+1$ colors. To achieve this, a simple greedy algorithm suffices:

For each vertex $P_{i}, i=1 \ldots 2^{n}$, choose for $f_{n}\left(P_{i}\right)$ the smallest value of $Z_{n+1}$ not belonging to the set $\left\{f_{n}\left(P_{j}\right): 1 \leq j<i\right\}$ and such that $\left(f_{n}\left(P_{i-1}\right) \oplus f_{n}\left(P_{i}\right)\right) \notin\left\{f_{n}\left(P_{j}\right) \oplus f_{n}\left(P_{j}+1\right)\right.$ : $1 \leq j<i-1\}$.

We can guarantee the existence of such a value at each step $i$ of this algorithm since:

- there are at least $2^{n}+1$ values available in $Z_{n+1}$ that do not belong to $\left\{f_{n}\left(P_{j}\right): 1 \leq\right.$ $j<i\}$.

- there are at most $2^{n}-2$ forbidden values in $\left\{f_{n}\left(P_{j}\right) \oplus f_{n}\left(P_{j}+1\right): 1 \leq j<i-1\right\}$, and there cannot be two distinct values $v_{1}, v_{2} \in Z_{n+1}$ such that $f_{n}\left(P_{i-1}\right) \oplus v_{1}=$ $f_{n}\left(P_{i-1}\right) \oplus v_{2}$.

Now see how the CP-transversal problem is connected to the determination of a minimum set coloring in a path of order $2^{n}-1$. Let $T$ be a CP-transversal of $A_{n}$ according to Definition 3 (the function $\Gamma$ is supposed to be defined). Let $P$ be a path of order $2^{n}-1$ with $n \geq 2$. We build a set coloring $\left(f_{n}, f_{n}^{\oplus}\right)$ of $P$ in the following way: if $(0,0,0) \notin T$, then $f_{n}\left(P_{1}\right)=0$, otherwise $f_{n}\left(P_{1}\right)=1$. Then for all $2 \leq i \leq 2^{n}-1$, we set $f_{n}\left(P_{i}\right)=\Gamma\left(f_{n}\left(P_{i-1}\right)\right)$.

Figures 6 and 7 illustrate this construction on paths of orders 7 and 15, using the CP-transversals $T_{3}$ and $T_{4}$ (depicted on Figures 4 and 5 ).

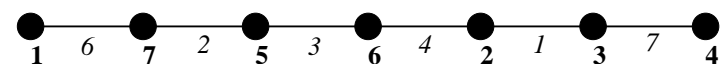

Figure 6: 3 -set coloring of a path of order 7 from $T_{3}$

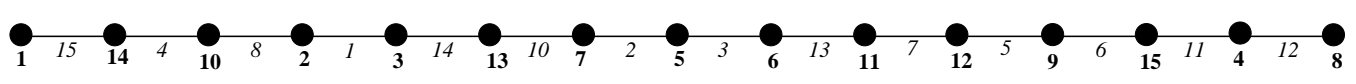

Figure 7: 4-set coloring of a path of order 15 from $T_{4}$

The validity of this construction can be explained as follows: since $\Gamma$ is a circular permutation of size at least $2^{n}-1$ over $Z_{n}$, we have the guarantee that the values $f_{n}\left(P_{i}\right)$ for $1 \leq i \leq 2^{n}-1$ are all distinct and belong to $Z_{n}$. Moreover, the values $f_{n}^{\oplus}\left(P_{i}, P_{i+1}\right)$ for $i=1, \ldots, 2^{n}-2$ correspond to the values of the cells of $T$. Hence they also belong to $Z_{n}$ and are all distinct. 
Remark 1. This proves that there exists no CP-transversal of $A_{n}$ with $\Gamma$ of size $2^{n}$. Indeed, if such a transversal exists, we could build a set coloring of size $n$ of a path of order $2^{n}$, contradicting Lemma 1 .

From the previous considerations, the following conjecture can be considered as a corollary of Conjecture 1 .

Conjecture 2. Given any positive integer $n \geq 2$, a path $P$ of order $2^{n}-1$ satisfies $\sigma(P)=n$.

Therefore, it turns out that a proof of Conjecture 1 would solve the set coloring problem on paths of any order, as detailed in Corollary 1.

Corollary 1. Given a path $P=(V, E)$ with $|V| \geq 3$, we have $\sigma(P)=\left\lceil\log _{2}(|V|+1)\right\rceil$.

Proof. The proof directly derives from Proposition 2 and Conjecture 2. Note that one also need to consider the monotonicity of the function $\Sigma: k \mapsto \sigma\left(P^{k}\right)$, where $P^{k}$ is the path of order $k$.

\subsection{Set coloring number of complete binary trees}

The complete binary tree of height $n>0$ will be denoted by $B T_{n}$. Note that $B T_{n}$ has exactly $2^{n}-1$ vertices. By using Proposition 1 about transversals of $A_{n}$, we will show that a complete binary tree has the smallest possible coloring number, i.e., $\sigma\left(B T_{n}\right)=n$.

Theorem 1. The complete binary tree $B T_{n}$ satifies $\sigma\left(B T_{n}\right)=n$ for all $n \geq 1$.

Figure 8 shows a strong 3 -set coloring of $B T_{3}$.

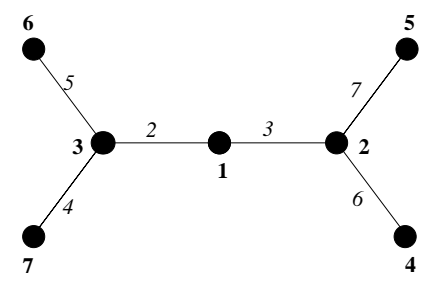

Figure 8: 3-set coloring of $B T_{3}$

Figures 9 and 10 illustrate the strong set coloring scheme detailed in the proof of Theorem 1. As it is easier to understand, we added the binary versions of the strong set colorings. Roughly speaking, one can explain this inductive technique as follows: the non-leaves vertices of $B T_{n}$ have the same color as the vertices of $B T_{n-1}$. If $(x, y)$ and $(x, z)$ are two edges of $B T_{n}$ such that $y$ and $z$ are two leaves, then $y$ and $z$ take the color $10 \Gamma(u)$ and $11 \Gamma(u)$ (in binary), where $u$ is the color of $x$ without its two most significant bits, and $\Gamma$ is the function corresponding to a transversal of $A_{n-2}$ (see Definition 3). 

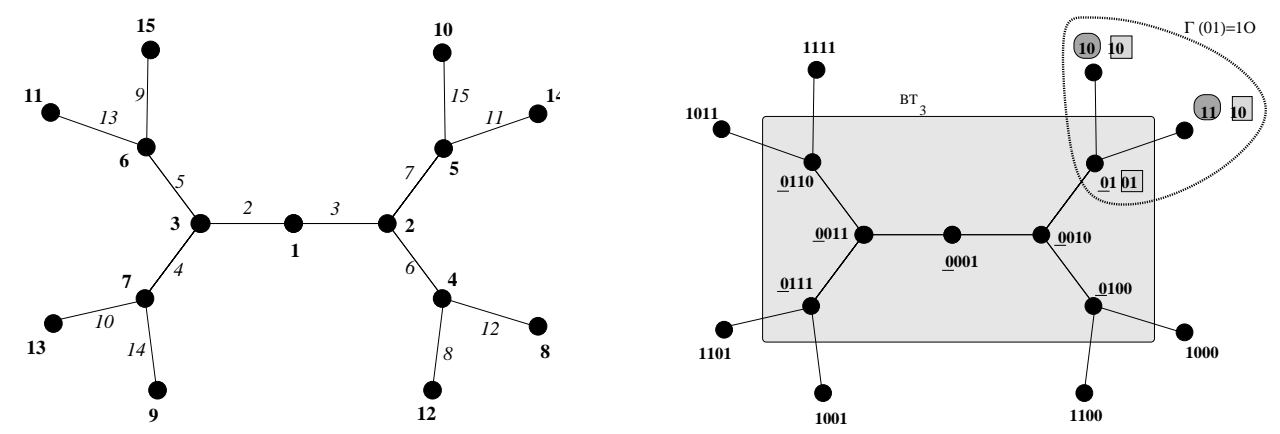

Figure 9: 4-set coloring of $\mathrm{BT}_{4}$ (decimal version on the left, and the equivalent binary version on the right), where $\Gamma$ is the one of the transversal $T_{2}$ of Figure 4

Proof. The theorem is clearly true for $n=1,2$. We thus consider $n \geq 3$.

We denote by $W_{n}$ the leaves of $B T_{n}=\left(V_{n}, E_{n}\right)$. Given any set coloring function $f_{n}: V_{n} \longrightarrow Z_{n} \backslash\{0\}$, we define $f_{n}^{\prime}: W_{n} \longrightarrow Z_{n-1}$ as $f_{n}^{\prime}: u \mapsto f_{n}(u) \bmod 2^{n-1}$. We now introduce the following induction hypothesis:

$\left(H_{n}\right)$ There exists a set coloring $\left(f_{n}, f_{n}^{\oplus}\right)$ of $B T_{n}$ with $n$ colors such that $f_{n}$ is a bijection from $V_{n}$ to $Z_{n} \backslash\{0\}$, and $f_{n}^{\prime}$ is a bijection from $W_{n}$ to $Z_{n-1}$.

According to Figure 8, Proposition $\left(H_{3}\right)$ is true. Now assume that $\left(H_{n}\right)$ is true for some $n \geq 3$. For more convenience, we will denote by $X=\left(V_{n+1}, E_{n+1}\right)$ the graph $B T_{n+1}$ and by $Y=\left(V_{n}, E_{n}\right)$ the graph $X \backslash W_{n+1}$. Notice that $Y=B T_{n}$. Since $Y$ satisfies $\left(H_{n}\right)$, there exists a set coloring $\left(f_{n}, f_{n}^{\oplus}\right)$ of $Y$ such that $f_{n}^{\prime}$ is a bijection from $W_{n}$ to $Z_{n-1}$.

Let $T$ be a transversal of $A_{n-1}$, equipped with the $\Gamma$ function given by Definition 3. According to Proposition 1, such a $T$ exists. For all $u \in W_{n}$, we denote by $L_{u}=$ $\left\{v \in W_{n+1}:(u, v) \in E_{n+1}\right\}$. Remark that we always have $\# L_{u}=2$. We then build the following set coloring $\left(f_{n+1}, f_{n+1}^{\oplus}\right)$ of $X$ :

- For all $x$ in $V_{n}$, we set $f_{n+1}(x)=f_{n}(x)$.

- For all $x$ in $W_{n}$, we set $f_{n+1}\left(L_{x}\right)=\left\{\Gamma\left(f_{n}^{\prime}(x)\right)+2^{n}, \Gamma\left(f_{n}^{\prime}(x)\right)+2^{n}+2^{n-1}\right\}$.

To prove that this coloring satisfies $\left(H_{n+1}\right)$, we proceed in three steps:

- The function $f_{n+1}^{\prime}$ is a bijection from $W_{n+1}$ to $Z_{n}$.

By way of contradiction, assume that there exist $x, y$ in $W_{n+1}$ such that $\# f_{n+1}^{\prime}(\{x, y\})=1$. We consider two cases:

(i) There exists $z$ in $W_{n}$ such that $x \in L_{z}$ and $y \in L_{z}$. Hence we get

$$
f_{n+1}(\{x, y\})=\left\{\Gamma\left(f_{n}^{\prime}(z)\right)+2^{n}, \Gamma\left(f_{n}^{\prime}(z)\right)+2^{n}+2^{n-1}\right\} .
$$



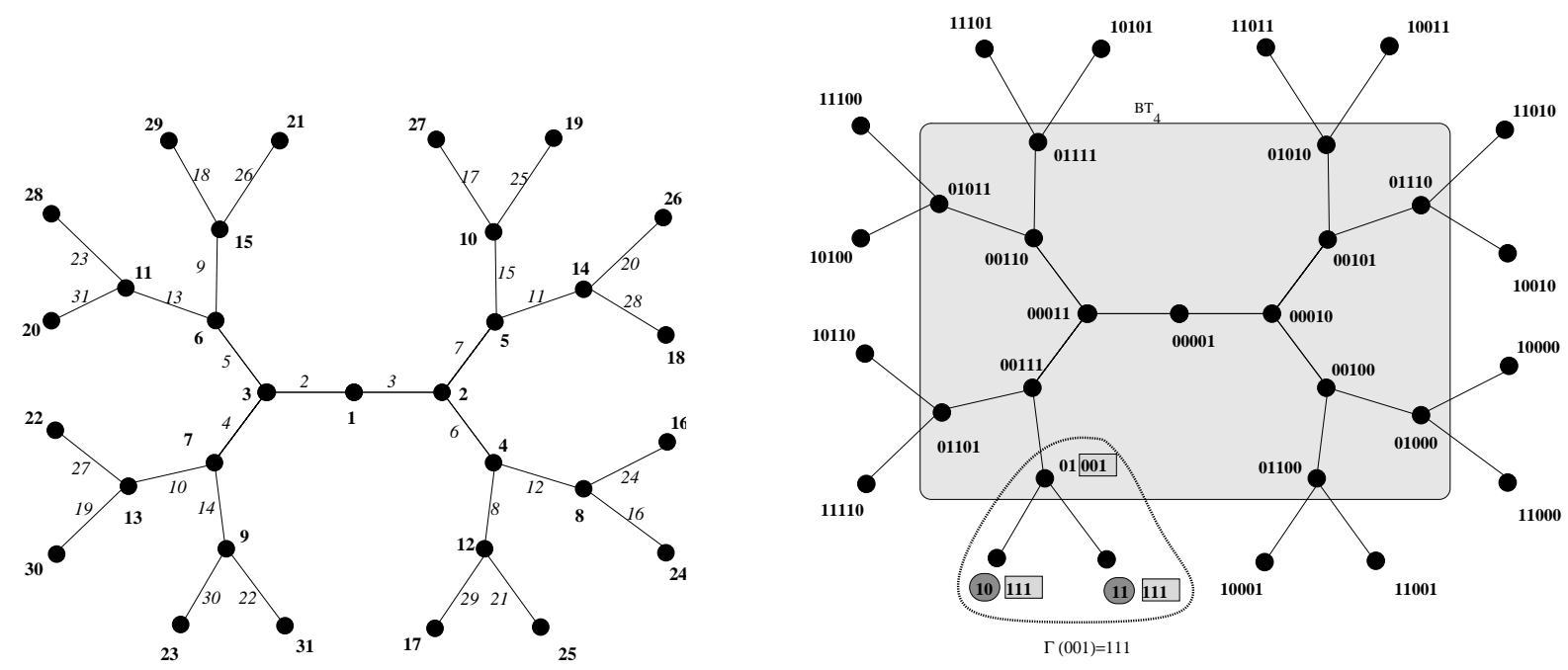

Figure 10: 5-set coloring of $B T_{5}$ (decimal version on the left, and the equivalent binary version on the right), where $\Gamma$ is the one of the transversal $T_{3}$ of Figure 4

Since $0 \leq \Gamma\left(f_{n}^{\prime}(z)\right) \leq 2^{n-1}-1$, we obtain

$$
f_{n+1}^{\prime}(\{x, y\})=f_{n+1}(\{x, y\}) \bmod 2^{n}=\left\{\Gamma\left(f_{n}^{\prime}(z)\right), \Gamma\left(f_{n}^{\prime}(z)\right)+2^{n-1}\right\},
$$

which implies $\# f_{n+1}^{\prime}(\{x, y\})=2$.

(ii) There exist $z, t$ in $W_{n}$ such that $x \in L_{z}$ and $y \in L_{t}$. With the same reasoning as in $(i)$, we get

$$
\begin{gathered}
f_{n+1}^{\prime}(x) \in\left\{\Gamma\left(f_{n}^{\prime}(z)\right), \Gamma\left(f_{n}^{\prime}(z)\right)+2^{n-1}\right\} \\
f_{n+1}^{\prime}(y) \in\left\{\Gamma\left(f_{n}^{\prime}(t)\right), \Gamma\left(f_{n}^{\prime}(t)\right)+2^{n-1}\right\}
\end{gathered}
$$

Since $f_{n}^{\prime}$ is bijective and $\Gamma\left(f_{n}^{\prime}(z)\right) \neq \Gamma\left(f_{n}^{\prime}(t)\right)$ by the definition of a transversal, we also get

$$
\left\{\Gamma\left(f_{n}^{\prime}(z)\right), \Gamma\left(f_{n}^{\prime}(z)\right)+2^{n-1}\right\} \cap\left\{\Gamma\left(f_{n}^{\prime}(t)\right), \Gamma\left(f_{n}^{\prime}(t)\right)+2^{n-1}\right\}=\emptyset
$$

Hence $\# f_{n+1}^{\prime}(\{x, y\})=2$.

- The function $f_{n+1}$ is a bijection from $V_{n+1}$ to $Z_{n+1} \backslash\{0\}$.

By the definition of $f_{n+1}$, we have

$$
f_{n+1}\left(W_{n+1}\right)=\Gamma\left(f_{n}^{\prime}\left(W_{n}\right)\right)+2^{n} \cup \Gamma\left(f_{n}^{\prime}\left(W_{n}\right)\right)+2^{n}+2^{n-1} .
$$

Hence we get

$$
f_{n+1}^{\prime}\left(W_{n+1}\right)=\Gamma\left(f_{n}^{\prime}\left(W_{n}\right)\right) \cup \Gamma\left(f_{n}^{\prime}\left(W_{n}\right)\right)+2^{n-1}
$$


and

$$
f_{n+1}\left(W_{n+1}\right)=f_{n+1}^{\prime}\left(W_{n+1}\right)+2^{n} .
$$

Since $f_{n+1}^{\prime}$ is a bijection from $W_{n+1}$ to $Z_{n}$, we get $f_{n+1}\left(W_{n+1}\right)=Z_{n}+2^{n}=Z_{n+1} \backslash Z_{n}$. Moreover, since $f_{n+1}(x)=f_{n}(x)$ for all $x \in V_{n}$, and as $f_{n}$ satisfies $\left(H_{n}\right)$, we have that $f_{n+1}\left(V_{n}\right)=Z_{n} \backslash\{0\}$. Hence we get $f_{n+1}\left(V_{n+1}\right)=f_{n+1}\left(V_{n}\right) \cup f_{n+1}\left(W_{n+1}\right)=Z_{n+1} \backslash\{0\}$.

- The function $f_{n+1}^{\oplus}$ is a bijection from $E_{n+1}$ to $Z_{n+1} \backslash\{0\}$.

We first show that $f_{n+1}^{\oplus}$ is a bijection from $E_{n+1} \backslash E_{n}$ to $Z_{n+1} \backslash Z_{n}$.

Let $e=(x, y) \in E_{n+1} \backslash E_{n}$ with $x \in W_{n}$ and $y \in W_{n+1}$. By definition of $f_{n+1}$, we have that

$$
f_{n+1}^{\oplus}(e) \in f_{n+1}(x) \oplus\left\{\Gamma\left(f_{n}^{\prime}(x)\right)+2^{n}, \Gamma\left(f_{n}^{\prime}(x)\right)+2^{n}+2^{n-1}\right\} .
$$

Hence $f_{n+1}^{\oplus}\left(E_{n+1} \backslash E_{n}\right) \subseteq Z_{n+1} \backslash Z_{n}$. Now suppose that there exist $e_{1}, e_{2} \in E_{n+1} \backslash E_{n}$ such that $\# f_{n+1}^{\oplus}\left(\left\{e_{1}, e_{2}\right\}\right)=1$. We consider two cases:

(i) $\exists(x, y, z) \in W_{n} \times W_{n+1} \times W_{n+1}$ such that $e_{1}=(x, y)$ and $e_{2}=(x, z)$. Then

$$
f_{n+1}^{\oplus}\left(\left\{e_{1}, e_{2}\right\}\right)=f_{n+1}(x) \oplus\left\{\Gamma\left(f_{n}^{\prime}(x)\right)+2^{n}, \Gamma\left(f_{n}^{\prime}(x)\right)+2^{n}+2^{n-1}\right\}
$$

Hence $\# f_{n+1}^{\oplus}\left(\left\{e_{1}, e_{2}\right\}\right)=2$, which contradicts the hypothesis.

(ii) $\exists(w, x, y, z) \in W_{n} \times W_{n+1} \times W_{n} \times W_{n+1}$ with $w \neq y$ and such that $e_{1}=(w, x)$ and $e_{2}=(y, z)$. Then

$$
\begin{gathered}
f_{n+1}^{\oplus}\left(e_{1}\right) \in f_{n+1}(w) \oplus\left\{\Gamma\left(f_{n}^{\prime}(w)\right)+2^{n}, \Gamma\left(f_{n}^{\prime}(w)\right)+2^{n}+2^{n-1}\right\} \\
f_{n+1}^{\oplus}\left(e_{2}\right) \in f_{n+1}(y) \oplus\left\{\Gamma\left(f_{n}^{\prime}(y)\right)+2^{n}, \Gamma\left(f_{n}^{\prime}(y)\right)+2^{n}+2^{n-1}\right\}
\end{gathered}
$$

We therefore get

$$
\begin{aligned}
f_{n+1}^{\oplus}\left(e_{1}\right) \bmod 2^{n-1} & =f_{n+1}(w) \bmod 2^{n-1} \oplus \Gamma\left(f_{n}^{\prime}(w)\right) \\
& =f_{n}^{\prime}(w) \oplus \Gamma\left(f_{n}^{\prime}(w)\right)
\end{aligned}
$$

and

$$
\begin{aligned}
f_{n+1}^{\oplus}\left(e_{2}\right) \bmod 2^{n-1} & =f_{n+1}(y) \bmod 2^{n-1} \oplus \Gamma\left(f_{n}^{\prime}(y)\right) \\
& =f_{n}^{\prime}(y) \oplus \Gamma\left(f_{n}^{\prime}(y)\right)
\end{aligned}
$$

Moreover, since all the squares of a transversal have a distinct value, we have:

$$
f_{n}^{\prime}(w) \oplus \Gamma\left(f_{n}^{\prime}(w)\right) \neq f_{n}^{\prime}(y) \oplus \Gamma\left(f_{n}^{\prime}(y)\right)
$$

and thus $\# f_{n+1}^{\oplus}\left(\left\{e_{1}, e_{2}\right\}\right)=2$, contradicting the hypothesis.

Consequently we get $f_{n+1}^{\oplus}\left(E_{n+1} \backslash E_{n}\right)=Z_{n+1} \backslash Z_{n}$. Moreover, since $f_{n}^{\oplus}$ satisfies $\left(H_{n}\right)$, we have that $f_{n+1}^{\oplus}\left(E_{n}\right)=f_{n}^{\oplus}\left(E_{n}\right)=Z_{n} \backslash\{0\}$. Hence $f_{n+1}^{\oplus}\left(E_{n+1}\right)=Z_{n+1} \backslash\{0\}$. 


\section{$3 \quad$ Strong set coloring}

Despite the proximity of their definitions, the approach of the strong set coloring problem is far different from the computation of the set coloring number of a graph. Thus, although the optimization of such a coloring is important, its existence is linked to the additional constraints it considers. Indeed, the set of graphs that are concerned by a strong set coloring is restricted, since they must satisfy $|V|+|E|=2^{n}-1$ for some $n$ as a preliminary condition. In that context, Hegde first proposed several results helping to cope with the strong set coloring of paths, complete graphs, complete bipartite graphs and complete binary trees.

\subsection{Previous results}

In his paper [4], Hegde gave a set of necessary conditions for a graph $G$ to be strongly set colorable. We here mention one his most relevant result that will be used further.

Proposition 3 (Hegde). If a graph $G$ of order $>2$ has:

(i) exactly one or two vertices of even degree or

(ii) exactly three vertices of even degree, say $v_{1}, v_{2}, v_{3}$, and any two of these vertices are adjacent or

(iii) exactly four vertices of even degree, say $v_{1}, v_{2}, v_{3}, v_{4}$ such that $\left(v_{1}, v_{2}\right)$ and $\left(v_{3}, v_{4}\right)$ are edges in $G$,

then $G$ is not strongly set colorable.

With the help of Proposition 3, Hegde proved the following results on existence and non-existence of strong set colorings:

Proposition 4 (S.M.Hegde). We have:

- the complete graph $K_{n}$ is strongly set colorable iff $n=2,5$.

- the complete $k$-ary tree is strongly set colorable iff it is a star $K_{1,2^{\alpha}-1}$ for some positive integer $\alpha$.

- the complete bipartite graph $K_{a, b}$ is strongly set colorable iff $(a+1)(b+1)=2^{\alpha}$ for some positive integer $\alpha$.

Moreover, Hegde conjectured that no path of order greater than 2 is strongly set colorable. The result is proved for paths of lengths 4,8,16.

\section{$3.2 \quad$ Other properties}

In addition to the results of Hegde, we here propose three other necessary conditions for a graph $G$ to be strongly set colorable, which are based on the existence of a special dominating set in $G$. As we will see further, these conditions turn out to be very efficient on small graphs, when trying to prove the non-existence of strong set colorings. 
Lemma 2. Let $G=(V, E)$ be a graph. If there exists a stable dominating set $D=\{u, v\}$ of size 2 such that for each edge $(w, z) \in E$ with $w, z \notin D$, we have $w \sim u$ and $z \sim v$ (or the opposite $w \sim v$ and $z \sim u$ ), then $G$ is not strongly set colorable.

Proof. Assume that there exists a strong set coloring $\left(f, f^{\oplus}\right)$ of $G=(V, E)$ and such a stable dominating set $D$. Hence the value $f(u) \oplus f(v) \in f(V) \cup f^{\oplus}(E)$. We consider the following cases for $f(u) \oplus f(v)$ :

- $f(u) \oplus f(v)$ is equal to $f(u)$ or $f(v)$. This would imply $f(u)$ or $f(v)$ equal to 0 , contradicting the definition of a strong set coloring.

- $f(u) \oplus f(v)=f(w)$ for some vertex $w \notin D$. Since $D$ is a dominating set, we have $w \sim u$ or $w \sim v$. Without loss of generality, assume that $w \sim u$. Hence we have $f^{\oplus}(u, w)=f(u) \oplus f(w)=f(v)$, which contradicts the strong set coloring condition.

- $f(u) \oplus f(v)=f^{\oplus}(u, w)$ for some vertex $w \notin D$. Hence we get the contradiction $f(v)=f(w)$.

- $f(u) \oplus f(v)=f^{\oplus}(v, w)$ for some vertex $w \notin D$. Similar to the previous case.

- $f(u) \oplus f(v)=f^{\oplus}(w, z)$ for some vertices $w, z \notin D$. Then by hypothesis, assume WLOG that we have $w \sim u$ and $z \sim v$. We therefore get the following contradiction:

$$
f^{\oplus}(w, u)=f(w) \oplus f(u)=f(u) \oplus f(v) \oplus f(z) \oplus f(u)=f(v) \oplus f(z)=f^{\oplus}(v, z) .
$$

Remark 2. Note that the conditions of Lemma 2 remain true to prove that a graph $G$ is not properly colorable.

Lemma 3. Let $G=(V, E)$ be a graph. If $G$ admits a dominating set $D$ such that $D$ is a clique of size 3 and $G \backslash D$ is a stable set, then $G$ is not strongly set colorable.

Proof. Assume that there exists a strong set coloring $\left(f, f^{\oplus}\right)$ of $G=(V, E)$ and such a stable dominating set $D=\{u, v, w\}$. We consider the following cases for $f(u) \oplus f(v) \oplus$ $f(w) \neq 0$ :

- $f(u) \oplus f(v) \oplus f(w)$ belongs to $f(D)$. This would imply $f^{\oplus}(e)=0$ for some edge $e \in\{(u, v),(v, w),(u, w)\}$.

- $f(u) \oplus f(v) \oplus f(w)=f(z)$ for some vertex $z \notin D$. Since $D$ is a dominating set, assume WLOG that $z \sim u$. Hence $f^{\oplus}(z, u)=f^{\oplus}(v, w)$ since $v \sim w$. This contradicts the definition of a strong set coloring.

- $f(u) \oplus f(v) \oplus f(w)=f^{\oplus}(e)$ for some edge $e \in\{(u, v),(v, w),(u, w)\}$. We quickly get a contradiction. 
- $f(u) \oplus f(v) \oplus f(w)=f^{\oplus}(y, z)$ for some vertices $y \in D$ and $z \notin D$. Assume WLOG that $y=u$. Hence we get $f(z)=f(v) \oplus f(w)=f^{\oplus}(v, w)$, leading to a contradiction.

Lemma 4. Let $G=(V, E)$ be a graph with a strong set coloring $\left(f, f^{\oplus}\right)$. If $G$ admits a stable set $D=\{u, v\}$ of size 2 and an edge $e=(w, z) \in E$ with $w, z \notin D$ such that $f(u) \oplus f(v)=f^{\oplus}(e)$ then $G \backslash e \cup\{u, v\}$ is strongly set colorable.

Proof. Assume that such a $D$ exists, and that $G$ has strong set coloring $\left(f, f^{\oplus}\right)$. Therefore we straightforwardly get a strong set coloring of $G \backslash\{(w, z)\} \cup\{(u, v)\}$, by copying $\left(f, f^{\oplus}\right)$, where the color of $(u, v) \in G \backslash\{(w, z)\} \cup\{(u, v)\}$ is the same as the color of $(w, z) \in G$.

Corollary 2. Let $G=(V, E)$ be a graph. If $G$ admits a stable dominating set $D=\{u, v\}$ of size 2 that does not satisfy the condition of Lemma 2 , and if for all $e=(w, z) \in E$ with $w, z \notin D$ and $(w, z \not u$ or $w, z \nsim v)$ we have $G \backslash e \cup\{u, v\}$ which is not strongly set colorable, then $G$ is not strongly set colorable.

Proof. According to the proof of Lemma 2, we necessarily have $f(u) \oplus f(v)=f^{\oplus}(w, z)$ for some vertices $w, z \notin D$ and either $w, z \not u$ or $w, z \nsim v$. We conclude with Lemma 4 .

We will now show how Proposition 3 and Lemmas 2,3,4 are a collection of tools that are sufficient to decide which graphs are strongly set colorable with 3 and 4 colors.

\subsection{Characterization of strongly 4-set colorable graphs}

Proposition 3 is enough to show that there exists a unique strongly 2-set colorable graph (which is $K_{2}$ ), and a unique strongly 3 -set colorable graph (which is $K_{1,4}$ ). The above results are useful to characterize the set of strongly 4 -set colorable graphs.

Theorem 2. Graphs that are strongly 4-set colorable are those given by Figure 12.

Proof. When dealing with strongly 4-set colorable graphs, there are four possibilities concerning the pair $(|V|,|E|)$ (we recall that the graphs are supposed simple and connected).

$|V|=5$ and $|E|=10$. A unique simple directed graph belongs to this category: $K_{5}$, which is strongly set colorable according to Proposition 4.

$|V|=6$ and $|E|=9$. The application of Proposition 3 leaves 9 graphs suitable for a strong set coloring. Among them, 6 are proved to be not colorable thanks to Lemmas 2 and 3. The 3 remaining graphs are strong set colorable, as shown on Figure 12 (b), (c) and (d).

$|V|=7$ and $|E|=8$. The application of Proposition 3, Lemmas 2, 3, 4 and Corollary 2 leaves 18 graphs suitable for a strong set coloring. Nine out of them are strongly set colorable (see the colorations (e) to (m) on Figure 12). For the nine other cases, we 
need more particular proofs based on the following technique: given $\left(f, f^{\oplus}\right)$ a strong set coloring of a graph $G$, we take a non null sum of $f\left(v_{i}\right)$ for some vertices $v_{i} \in V$ and see where it can be located in the strong set coloring. The objective is to get contradictions by using Lemma 4 and the fact that $\Sigma f\left(v_{i}\right)=0$ for all $v_{i} \in V$ of even degree. We illustrate this technique to prove that the graph (a) of Figure 11 is not strongly set colorable.

We consider a strong set coloring $\left(f, f^{\oplus}\right)$ of the graph (a) in Figure 11 and the sum

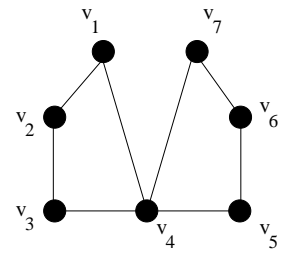

a)

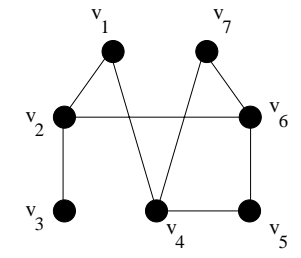

b)

Figure 11: Example of a non strongly set colorable graph

$f\left(v_{2}\right) \oplus f\left(v_{3}\right) \oplus f\left(v_{4}\right) \oplus f\left(v_{5}\right)$ which is non zero since $\left(v_{2}, v_{3}\right),\left(v_{4}, v_{5}\right) \in E$. We distinguish two cases.

- $f\left(v_{2}\right) \oplus f\left(v_{3}\right) \oplus f\left(v_{4}\right) \oplus f\left(v_{5}\right)=f\left(v_{5}\right)+f\left(v_{6}\right)$. Hence we have $f\left(v_{3}\right) \oplus f\left(v_{4}\right)=$ $f\left(v_{2}\right)+f\left(v_{6}\right)$. In view of Lemma 4, this implies that the graph (b) of Figure 11 is also strongly set colorable. This is not the case from Lemma 3.

- $f\left(v_{2}\right) \oplus f\left(v_{3}\right) \oplus f\left(v_{4}\right) \oplus f\left(v_{5}\right) \neq f\left(v_{5}\right)+f\left(v_{6}\right)$. We straightforwardly find a contradiction with the definition of the strong set coloring, possibly by using the equality $\sum_{i=1}^{7} f\left(v_{i}\right)=0$.

$|V|=8$ and $|E|=7$. Such graphs are trees. There are only five trees that do not satisfy the conditions of Proposition 3. Four out of five are strongly 4-set colorable (see the colorations (n),(o),(p) and (q) on Figure 12). The last one is the path $P_{8}$, which is not strongly 4-set colorable. Indeed, assume there exists a strong set coloring $\left(f, f^{\oplus}\right)$ of $P_{8}$. Hence we get $\sum_{i=1}^{8} f\left(P_{i}\right)+\sum_{i=1}^{7} f^{\oplus}\left(P_{i}, P_{i+1}\right)=\sum_{i=2}^{6} f\left(P_{i}\right)=0$, which can also be written as

$$
f\left(P_{2}\right)+f\left(P_{3}\right)+f\left(P_{4}\right)=f\left(P_{5}\right)+f\left(P_{6}\right)+f\left(P_{7}\right)
$$

We consider the following cases for $f\left(P_{2}\right)+f\left(P_{3}\right)+f\left(P_{4}\right)$ :

- $f\left(P_{2}\right)+f\left(P_{3}\right)+f\left(P_{4}\right)=f\left(P_{i}\right)$ for some $i$ in $1 \ldots 8$. Whatever the value of $i$ and according to $(*)$, we quickly get a contradiction with the definition of a strong set coloring.

- $f\left(P_{2}\right)+f\left(P_{3}\right)+f\left(P_{4}\right)=f\left(P_{i}\right)+f\left(P_{i+1}\right)$ for some $i$ in $1 \ldots 7$. For the same reasons, the partition constraint of a strong set coloring cannot be fulfilled whatever the value of $i$. 


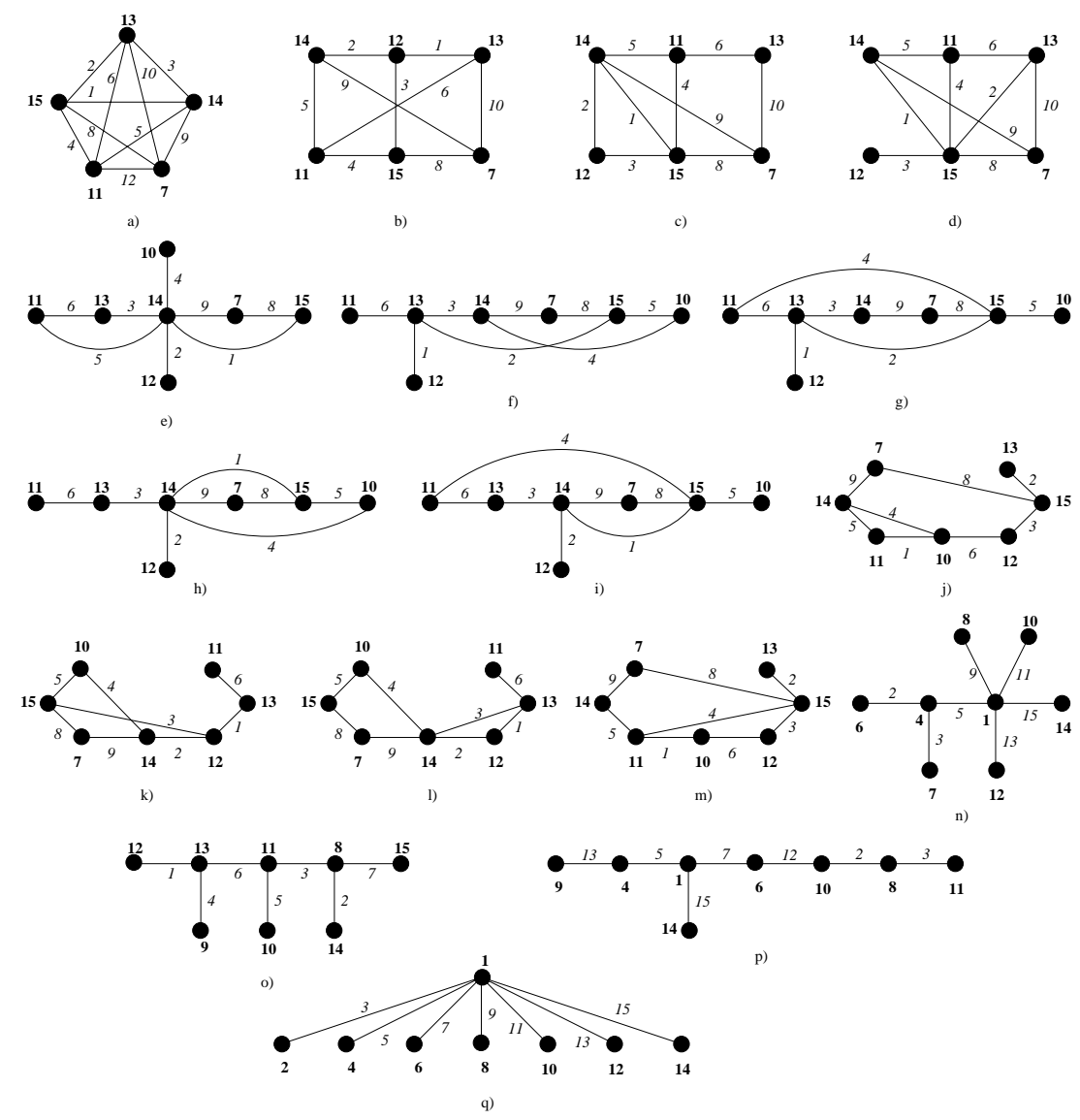

Figure 12: Graphs with a strong 4-set coloring

Remark 3. In order not to make the paper heavier, we did not give all the sub-proofs of Theorem 2 in their entirety. Note that this theorem was also proved by a computer program.

\section{References}

[1] R.A. Brualdi and H.J. Ryser, Combinatorial matrix theory, Encyclopedia of Mathematics and its Applications, Cambridge (1991).

[2] J. Dénes and A.D. Keedwell, Latin squares: new developments in the theory and applications, Annals of Discrete Mathematics 46 (1991), North-Holland, New-York.

[3] E. Duchêne, A.S. Fraenkel, S. Gravier, R.J. Nowakowski, Another bridge between Nim and Wythoff, Australasian Journal of Combinatorics 44 (2009), 43-56.

[4] S.M. Hegde, Set colorings of graphs, European Journal of Combinatorics 30(4) (2009), 986-995.

[5] H.J. Ryser, Neuere Probleme der Kombinatorik, Vortrage über Kombinatorik Oberwolfach, Mathematisches Forschungsinstitut Oberwolfach (1967), 24-29. 